\title{
A cricket-inspired Neural Network For FeedForward Compensation and Multisensory Integration
}

\author{
Paolo Russo, Barbara Webb, Richard Reeve, Paolo Arena, Luca Patané
}

\begin{abstract}
A nonlinear feedforward compensator was designed as part of a bioinspired neural network to model sensorimotor integration and control in crickets. Female crickets perform auditory orientation (phonotaxis) towards the male's calling song to find a mate. Crickets also use visual sensing, for example in the optomotor reflex which allows them to maintain a straight trajectory against disturbances. The compensator describe in this paper allows the efficient integration of the phonotaxis and optomotor systems. The design is inspired by the neurophysiological concepts of efferent-copy and corollary discharge, which can be directly intepreted within control theory as feedforward compensation for predictable disturbances. The aim is to predict the reafferent visual stimulus caused by phonotaxis, based on the efferent response, thus filtering out the optical disturbances induced by the phonotactic reflex, while still detecting any external noise. The feedforward compensator design was formulated as an identification problem, drawing data from experiments on a robot performing phonotaxis. The compensator parameters were first derived by trial-and-error, and then optimised using a genetic algorithm. The scheme is implemented in a bioinspired neural network on a robot, and experiments are carried out to compare the behaviour to the cricket.
\end{abstract}

\section{INTRODUCTION}

The realization of bioinspired control schemes needs strong interaction between different disciplines such as control theory and neurophysiological studies. The auditory behaviour of the cricket has been extensively studied by biologists [1], [3], [4] and has been the subject of a series of robot models [2], [5]-[8]. In this paper we extend the robot, to test a neurally-implemented 'forward model' [9] as a solution to the control problem of combining auditory and visual behaviours.

Female crickets are able to recognise the species specific pattern of male calling song and move towards it. In recent work [10] new data from female crickets reveals a rapid steering response towards the male, modulated by recognition of the song pattern at a slower timescale. In [11] a neural system which includes the rapid reactive pathway and the slow perceptive recognition was designed and tested on a robot. This model is called the fast model since the acoustic stimulus gives rise to a reaction in $55-60 \mathrm{~ms}$. Details of the results from experiments comparing the resulting auditory behaviour to the cricket are provided in [11]. Here we focus

P.Arena, P.Russo and L.Patané are with the Dipartimento di Ingegneria Elettrica Elettronica e dei Sistemi, Universitá degli studi di Catania, Italy. email: parena@diees.unict.it

B.Webb and R.Reeve are with the Institute of Perception, Action and Behaviour, University of Edinburgh, UK. email: bwebbeinf.ed.ac.uk on the issue of adding a visual capability, the optomotor reflex to the robot.

For a mobile robot (or an animal) a rotation of the entire visual field is usually the result of self-rotation. Consequently, unintentional self-rotation can be corrected by turning in the opposite direction in response to visual rotation signals. This reflex compensates for external disturbances and inaccuracies of the muscle-motor response, to maintain a straight trajectory. Note, however, that the acoustic and visual mechanisms have conflicting aims. The auditory response tries to align the trajectory towards the sound source, while the visual response tries to correct for any change in the trajectory, thus counteracting the alignment attempted by the auditory system.

Different solutions have been proposed to solve this sensory conflict [7], [12], [14] including: adding the outputs of the visual and auditory systems with different gains; having the auditory system output inhibit the visual system output; having the auditory system output inhibit the visual system input; subtracting the auditory system output from the visual system input; and having the auditory system output control behaviour via the visual control system.A comparison among these methods, as discussed in [14], showed that simple suppression of one sensory system by the other was a reasonably effective mechanism. However none of these implementations used dynamics.

A more suitable control method is to predict the amplitude and time-evolution of the visual stimulation that arise when an auditory response is accomplished. It was first suggested by von Holst and Mittelstadt in 1950 that biological systems may use an internal efferent copy of motor command signals to modulate sensory processing for predicted reafference. A similar idea was simultaneously proposed by Sperry, i.e. that sensory areas receive a corollary discharge corresponding to the expected feedback. Although this concept is often referred to in biology, there is surprisingly little direct neurophysiological evidence of connections from motor to sensory systems that could support this function. Moreover, biologists often overlook the problem that the system must somehow calculate the expected feedback - in control theory terms, it must implement a forward model to be able to compensate the expected disturbance [9].

The control strategy proposed in this paper consists of a dynamic system able to predict the external visual influences due to the acoustically driven motor command. The prediction is used to inhibit the optomotor system, so that it is smoothly combined with phonotaxis. Moreover, the system is realised as part of a network of spiking neurons, 


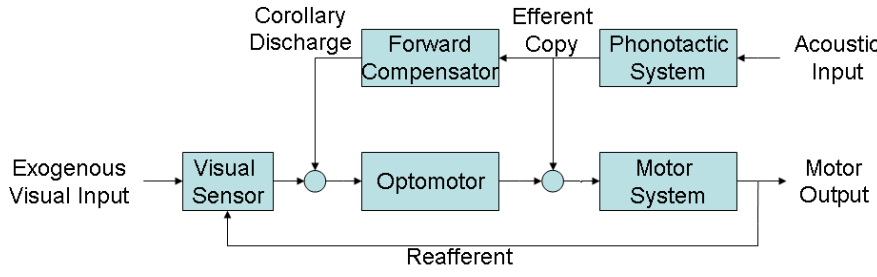

Fig. 1. The FeedForward compensation model.

demonstrating that it is a plausible solution for the cricket, and can be used effectively in controlling a mobile robot. We suggest that this methodology, used to integrate acoustic and visual stimuli, could be extended to many similar problems of integrating sensory stimuli.

\section{DESIGN OF THE CONTROL SYSTEM}

\section{A. Control theory basis and application}

The multisensory integration is based on feedforward compensation, a well-known method in industrial applications of control theory. It is an simple method to compensate for disturbances that are a priori known and accessible. In such cases, the correct identification of the plant allows an exact compensation of the noise. Here we consider the optomotor reflex as the plant and the phontaxis system as the source of noise: acoustic sensing produces a series of actions that will change the motor output planned by the optomotor reflex. Hence it can be compensated by using a dynamic feedforward model of the plant to predict the visual sensory (reafferent) signal corresponding to any action caused by the phonotaxis behaviour (Fig. 1). Each pair of phonotactic motor signals and the corresponding visual stimulus form an output-input couple, utilized to learn the required dynamic.

\section{B. Neural Network}

The neural architecture is based on the neuron models described by Koch in[15]. The membrane potential dynamics is represented by a simple first-order ordinary differential equation (ODE) and a spike occurs when a threshold is reached (i.e. an integrate and fire neuron) [16]. The state of the membrane $V_{m e m b}$ is governed by the following equation:

$$
V_{\text {memb }} \stackrel{\bullet}{\bullet}(t)+\frac{G_{m e m b}}{C_{m e m b}} V_{\text {memb }}(t)=\frac{G_{m e m b}}{C_{\text {memb }}} V_{\text {rest }}
$$

where the $G_{m e m b}, C_{m e m b}$ and $V_{\text {rest }}$ are respectively the conductance, the capacitance and the resting value of the neural cell. The neural equation is extended when the neuron is excited by a synapse. The synaptic contribute is visible in (2), where $G_{s y n_{i}}$ and $V_{s y n_{i}}$ are the conductance and the reference voltage potential of the synapse. The index $i$ is used because several synapses should be considered.

$$
\begin{gathered}
\stackrel{\bullet}{V_{\text {memb }}(t)}+\frac{\left(G_{m e m b}+\sum_{i} G_{\text {syn }}(t)\right)}{C_{m e m b}} V_{\text {memb }}(t)= \\
{\left[\left(V_{\text {rest }} G_{m e m b}\right)+\sum_{i}\left(V_{\text {syn }_{i}} G_{\text {syn }}(t)\right)\right] / C_{\text {memb }}} \\
V_{\text {axon }}(t)=0
\end{gathered}
$$

When the membrane potential reaches the threshold value $V_{t h}$ it is set to its recovery value $V_{\text {rec }}$ and kept for a refractory time $\left(T_{r e f}\right)$, thus a spike takes place, according to the following equation:

$$
i f\left(V_{\text {memb }}(t)>V_{t h}\right)\left\{\begin{array}{l}
V_{\text {axon }}(t)=1 \\
V_{\text {memb }}(t)=V_{\text {rec }}
\end{array}\right.
$$

Synaptic effects are modelled as changes in conductance, and include facilitation and depression mechanisms to allow temporal filtering. Such techniques allow the construction of small networks with useful capabilities, such as selectivity to particular patterns, or copying of external dynamics. The trend of conductance $G_{s y n}$, facilitation $G f a c_{s y n}$ and depression $G_{d e p_{s y n}}$ is exponential, each one with its characteristic exponential timing (respectively $T_{s y n}, T f a c_{s y n}$ and $\left.T d e p_{\text {syn }}\right)$. These variables are governed by the following first-order ODEs:

$$
\begin{aligned}
& G_{s y n}(t)+G_{s y n}(t) \frac{\ln 2}{T_{s y n}}=0 \\
& \operatorname{Gfac}_{s y n}(t)+G f a c_{s y n}(t) \frac{\ln 2}{T f a c_{s y n}}=0 \\
& \operatorname{Gdep}_{\text {syn }}(t)+\operatorname{Gdep}_{\text {syn }}(t) \frac{\ln 2}{\operatorname{Tdep}_{\text {syn }}}=\frac{\ln 2}{\operatorname{Tdep}_{\text {syn }}}
\end{aligned}
$$

Therefore the spikes emitted by the pre-synaptic neurons trigger the facilitation and depression mechanisms in order to produce the synaptic input $G_{s y n}$ for the post-synaptic neuron, as illustrated in 2 . When a spike occurs $\left(V_{\text {axon }}(t)=1\right)$, the synaptic variables are updated applying the algebraic equations described below:

$$
\begin{aligned}
& G_{s y n}(t)=G_{s y n}(t)+G d e p_{s y n}(t)\left[{G i n c_{s y n}}+G f a c_{s y n}(t)\right] \\
& \operatorname{Gfac}_{\text {syn }}(t)=\operatorname{Gfac}_{\text {syn }}(t)+\text { Gfacinc }_{\text {syn }} \\
& \operatorname{Gdep}_{\text {syn }}(t)=\operatorname{Gdep}_{\text {syn }}(t) \cdot G_{\text {depfrac }} \text { syn }
\end{aligned}
$$

where Ginc $_{\text {syn }}$ represents the increasing value of the synaptic conductance, Gfacinc $_{\text {syn }}$ the increasing value of the facilitation and Gdepfrac $_{\text {syn }}$ the fraction to which the depression variable is reduced.

The neuron model introduced in $(2,3)$ and the synapses described in $(4,5)$, have been used as elementary blocks to build a bioinspired neural network. The circuit was built to resemble the cricket neural system, and can be divided into 4 different subsystems (Fig. 2):

A: The optomotor reflex senses the left/right virtual movement of the world and consequently drives the motor controller neurons to compensate the visual stimuli.

B: The acoustic sensing is based on cricket physiology and is described in detail in [7].

C: The phonotactic reflex has two different pathways: the reactive one $(\mathrm{AN} 1 \rightarrow$ Fast $)$ and the perceptive one $(\mathrm{AN} 1 \rightarrow \mathrm{BN} 1 \rightarrow \mathrm{BN} 7 \rightarrow$ Gate $\rightarrow$ Fast $)$ [11].

D: The feedforward compensator has been designed to take the outgoing signal from the phonotactic system and to compensate the corresponding reafferent optomotor signal.

An internal source of noise is used to test the correct working of the optomotor reflex. The motor controller units are biased with random spikes, to represent disturbances that 


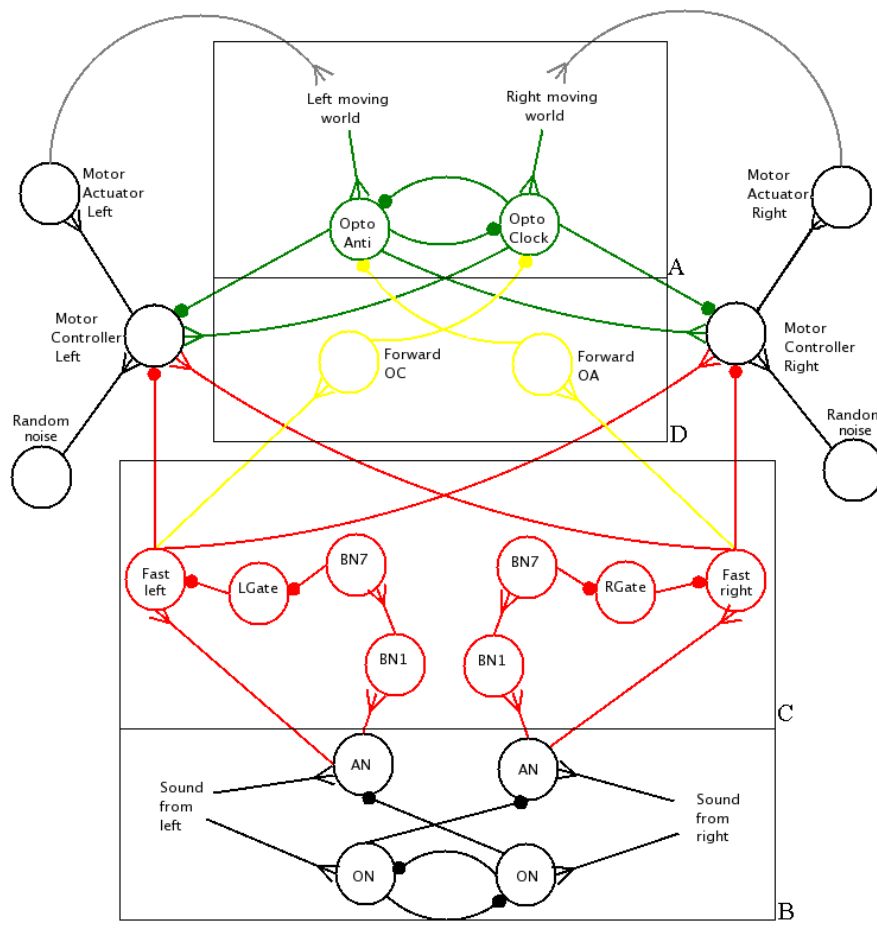

Fig. 2. Schema of the cricket bioinspired neural network model. The whole network is constituted by several subsystems: the optomotor reflex (substem A), the acoustic sensing block (subsystem B), the phonotactic model (subsystem C), the feedforward compensator (subsystem D), the motor controllers and an internal source of noise.

could occur during walking in crickets, due to asymmetries in the motor system or to external factors such as uneven terrain.

\section{FeedForward compensator design}

The feedforward compensator (i.e. subsystem $\mathrm{C}$ in Fig. 2) integrates the responses of the phonotactic system and the optomotor reflex. It takes the driving commands from the phonotactic system and tries to predict the reafferent visual signal. The driving commands also stimulate the motor controllers and consequently the motor actuators. A sound recognized, e.g. from the left, produces an inhibition on the left motor controller and an excitation on the right one, making the robot steer to left towards the sound source. There is a significant delay (a few hundred milliseconds) before the optomotor reflex will sense this left turn. Without any compensation, the optomotor system would react with a correction to the right side, driven by the opto-clockwise (OC) neuron, annihilating phonotaxis. The feedforward compensator (forward-OC neuron and related synapses) will avoid this incorrect response. The Fast-left neuron will stimulate the forward-OC neuron with the same number of spikes used to drive the motor controller. If the parameters of the forward-OC neuron and its input and output synapses are tuned to predict the reafferent signal, this will counterbalance the OC neuron stimulation, holding the neuron on its resting membrane potential.

Tuning of the forward neuron, pre- and post-neuron synapses was carried out initially by hand, and then using a genetic algorithm (GA) as an optimization strategy. In order to identify the feedforward compensator, data were gathered from experiments in which the roving Koala robot was allowed to control steering using only phonotaxis, i.e. with the synaptic connection of the optomotor system to the motors disabled. During these experiments the reafferent visual signals were recorded. As depicted in Fig. 3, we considered as input the efferent copy, i.e. the signal coming from the phonotactic system (Fig. 3(a)), and as output the reafferent signal, i.e. the opto neuron membrane potential (Fig. 3(b)-lower signal). This membrane potential change results from the changed conductance of the input synapse, Fig. 3(c), which represents the noise to be filtered.

Following the guidelines of classical identification, we assumed the feedforward model had a fixed dynamic structure, for which a number of parameters had to be optimally identified. The structure was fixed by the neuron/synapse structure already present into the complete model of Fig. 2. Moreover a subset of the parameters could be a priori fixed due to some preliminary considerations. For example, appropriate synaptic delays could be directly estimated from a correlation analysis between the efferent signal (cause) and the reafferent signal (effect). Following these considerations produced a forward nonlinear model structure constituted by 7 ODEs with 18 parameters to be optimised from experimental data.

The GA approach, as is well known, minimises a fitness function. The aim here is to keep the opto neuron to its resting potential, thus filtering out the phonotacticto-motor noise, so the fitness function was chosen as the squared-difference between the membrane potential of the opto neuron (Fig. 3(b)-lower signal) and its own resting value, when this neuron was excited by a signal due to the phonotactic behavior of the system. The neural parameters to be optimised are: the resting $\left(V_{\text {rest }}\right)$ and recovery $\left(V_{\text {rec }}\right)$ values of the membrane potential, the spiking threshold ( $\left.V_{t h}\right)$, the refractory time $\left(T_{r e f}\right)$, the membrane conductance $\left(G_{m e m b}\right)$ and capacitance $\left(C_{m e m b}\right)$. The synaptic parameters to optimise are: the conductance increasing value $\left(G i n c_{s y n}\right)$, the facilitation effect $\left(\right.$ Gfacinc $\left._{\text {syn }}\right)$, the depression fraction $\left(G_{\text {depfrac }} c_{\text {syn }}\right)$, the characteristic times of the conductance $\left(T_{\text {syn }}\right)$, the facilitation $\left(T f a c_{s y n}\right)$ and depression $\left(\right.$ Tdep $\left._{s y n}\right)$. The total number of parameters is 18 ( 6 for the neuron and 6 for each synapse) and we chose a precision of 50 bit for each parameter. The number of individuals was 200 and 1000 the number of generations. The generation gap was 0.9 , the mutation probability was 0.05 and 0.7 the recombination rate.

The results of the GA optimization stage are reported in Fig. 4. 4(a) shows the output (upper signal) and membrane potential (lower signal) of the optomotor neuron, when the optimised feedforward model is introduced into the loop. It can be seen that the optomotor neuron is completely inhibited during phonotactic behaviour. Fig. 4(b) shows the feedforward compensator output, i.e. the forward synapse conductance, while Fig. 4(c) depicts the reafferent optomotor synapse conductance. The obtained results confirm the suitability of the proposed control system. In fact, the forward 
(a)

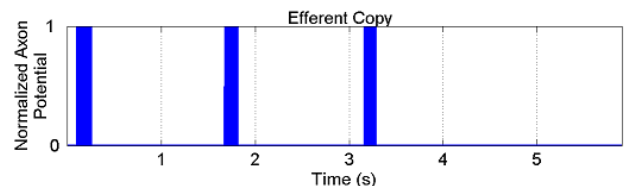

(b)

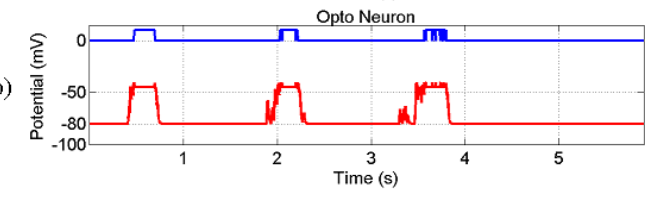

Reafferent Signal

(c)

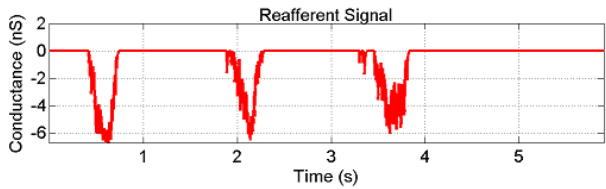

Fig. 3. Neural data gathered after a turn. It is shown: (a) the efferent copy signal coming from the phonotactic system; (b) the output (spike) of the opto neuron(upper signal) and the membrane potential (lower signal); (c) the conductance of the excitatory synapse coming from the visual sensor.

(a)

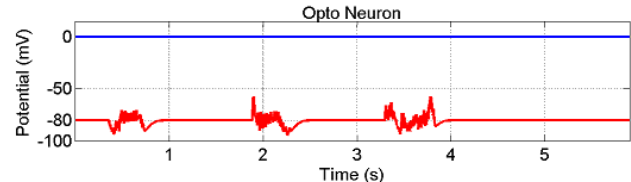

(b)

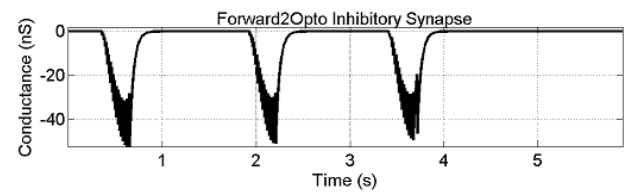

(c)

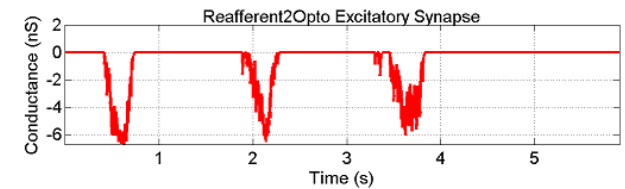

Fig. 4. Neural data with the forward compensator enabled. It is shown: (a) the output (spike) of the opto neuron(upper signal) and the membrane potential (lower signal); (b) the feedforward compensator output; (c) the conductance of the excitatory synapse coming from the visual sensor.

synapse dynamics (predicted signal), indirectly found by optimization, has the same shape as the reafferent optomotor synapse, so succeeds in balancing its effect. The two conductance scales are rather different because the reference voltage potential value for the excitatory synapse is $0 \mathrm{mV}$, whereas for the inhibitory synapse is $-100 \mathrm{mV}$. Since the resting value of the neuron is set on $-80 \mathrm{mV}$, the inhibition needs a larger conductance value to hold down the membrane potential of the neuron against excitation.

The results obtained by using the feedforward compensation allow concurrent use of both phonotactic and optomotor reflexes. The visual noise introduced by the phonotactic behavior is now efficiently filtered out without suppressing the optomotor system, which is ready to react to other external environment disturbance.

\section{Experimental setup}

The robot behaviour is tested in an indoor arena. The robot is a Koala, equipped with auditory and visual sensors based on insect sensory systems as described in [12]. A tether connects the robot to a PC running the neural simulation program and recording data. The auditory stimulus is a simulated cricket song. The speaker is placed on the laboratory floor. No special soundproofing or other controls for noise or echoes are used. We carry out trials with the robot starting in one of three positions: from near the center of the room, facing the speaker from about $180 \mathrm{~cm}$ and from half-way down each side wall, facing the opposite wall, about $160 \mathrm{~cm}$ from the speaker. For each position ten trials are recorded. The robot is stopped when it is about to hit the speaker or else one of the lab walls; a successful trial is counted when the center of the robot is within $30 \mathrm{~cm}$ of the speaker at the point it is stopped. The tracks are recorded using shaft encoders, which are sufficiently accurate for dead reckoning over the short paths considered in these experiments. The number of shaft encoder counts coming from the left and right wheels for each successive point recorded (usually every $100 \mathrm{~ms}$ ) is converted to $\mathrm{x}-\mathrm{y}$ position data.

To valuate the robot performance in each set of trials, a 'directness' parameter is calculated [17]:

From the $x-y$ coordinates we compute the distance covered and heading angle for each acquisition step:

$$
\begin{aligned}
\text { distance }_{i} & =\sqrt{\left(x_{i+1}-x_{i}\right)^{2}+\left(y_{i+1}-y_{i}\right)^{2}} \\
\text { heading }_{i} & =\operatorname{atan}\left(\frac{y_{i}}{x_{i}}\right)-\operatorname{atan}\left(\frac{y_{i}-y_{i+1}}{x_{i}-x_{i+1}}\right)
\end{aligned}
$$

The average vector for these moves is then calculated

$$
\begin{gathered}
\bar{x}=\frac{\sum \text { distance }_{i} \times \cos \left(\text { heading }_{i}\right)}{\sum \text { distance }_{i}} \\
\bar{y}=\frac{\sum-\text { distance }_{i} \times \sin \left(\text { heading }_{i}\right)}{\sum \text { distance }_{i}} \\
\text { angle }=\text { atan }\left(\frac{\bar{y}}{\bar{x}}\right) \\
\text { magnitude }=\bar{x}^{2}+\bar{y}^{2}
\end{gathered}
$$

The robot speed is used to calculate the tracktime:

$$
\text { tracktime }=\frac{\text { minimum time to do trial }}{\text { actual time to do trial }}
$$

The maximum speed of the robot is $200 \mathrm{~mm} / \mathrm{s}$. Finally we compute the directness:

$$
\text { directness }=\text { magnitude } \times \cos (\text { angle }) \times \text { tracktime }
$$

The range of the directness is within 0 and 1 , because all its factors are in that range. To explain the meaning of directness we can consider a route straight forward toward the source of the sound. In that case we have a null angle and a maximum amplitude, thus the directness value is one.

\section{EXPERIMENTS}

In this section we present the results of several experiments carried out to demonstrate the functioning of the system under different conditions. Note that the parameters used for the compensator in these experiments were those initially derived by hand-tuning. 
Phonotaxis: Fig. 5 shows the behaviour of the robot driven by phonotaxis only. It performs successfully,reaching the target every time, with directness $=0.38$.

Phonotaxis and Optomotor Reflex: Fig. 6 shows the trials carried out when the optomotor reflex is also enabled and a potential conflict with phonotaxis can occur. The robot does not manage to reach the target every time. The optomotor reflex allows the phonotaxis to work correctly when the angle towards the sound source small, in which case it helps to keep the robot stabilised in the correct direction, hence the directness is slightly higher overall $(=0.40)$.

Phonotaxis and Random Noise: The phonotaxis behaviour when a random noise is introduced into the motor output is shown in Fig. 7. The trajectories are more winding, but phonotaxis still guides the robot towards the loudspeaker, despite the noise, so the auditory behaviour is robust. The directness is lower (directness $=0.31$ ) than the default value.

Phonotaxis, Optomotor Reflex and Random Noise: Fig. 8 shows the trials carried out when both the random noise and the optomotor reflex are enabled, but the forward model is not active. The trajectories show the conflict between the two sensory systems. Although both the phonotaxis and optomotor systems are working against the noise, the optomotor reflex also "corrects" phonotactic turns. The directness is very low (directness $=0.22$ ).

FeedForward compensator allows to integrate Phonotaxis and Optomotor Reflex: The forward model behaviour is shown in Fig. 9. Here the feedforward controller compensates the reafferent signal, correctly predicting optical signals induced by phonotaxis. The optomotor reflex no longer tries to "correct" these turns, leaving the phonotaxis to reach the target. Directness is improved (directness $=0.44$ ) showing that the optomotor reflex is still active in stabilising the trajectory, but now without interfering with the phonotaxis. Multisensory integration with Random Noise In the final experiment the multisensory integration capabilities of

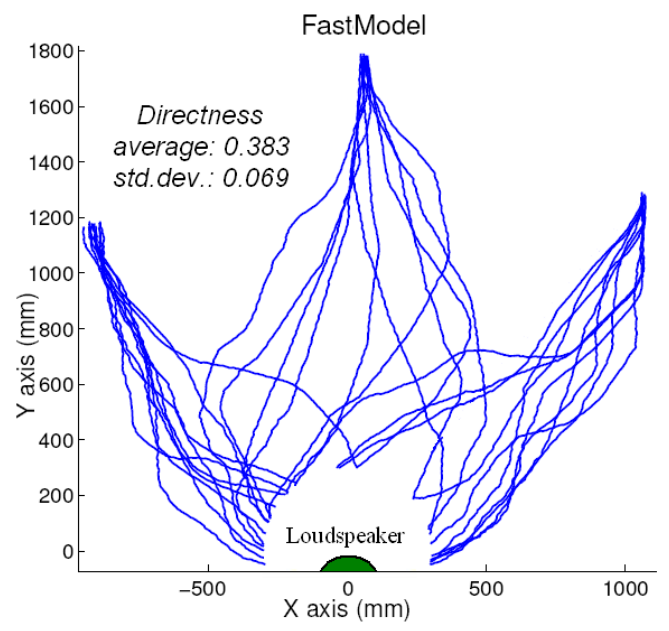

Fig. 5. Experimental results. The robot starts from three different positions. For each position ten trials are recorded. The robot every time reaches the target.

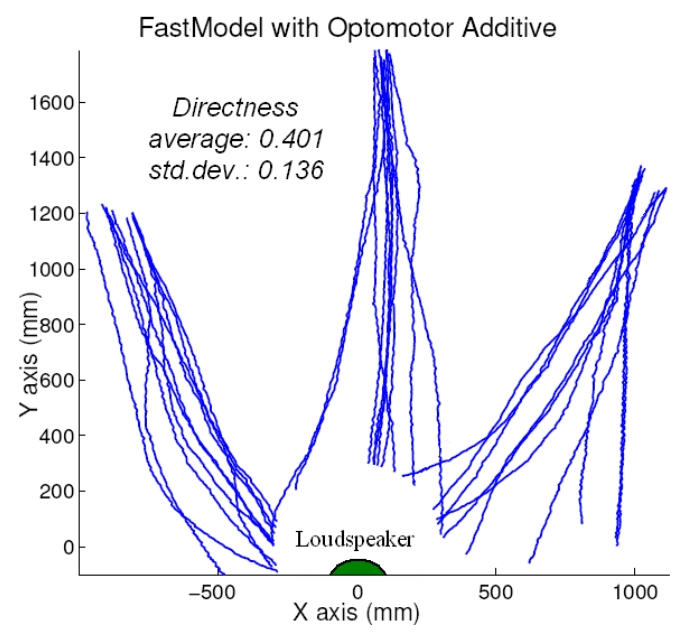

Fig. 6. Phonotaxis and optomotor are in additive mode. The robot sometimes is not able to reach the target.

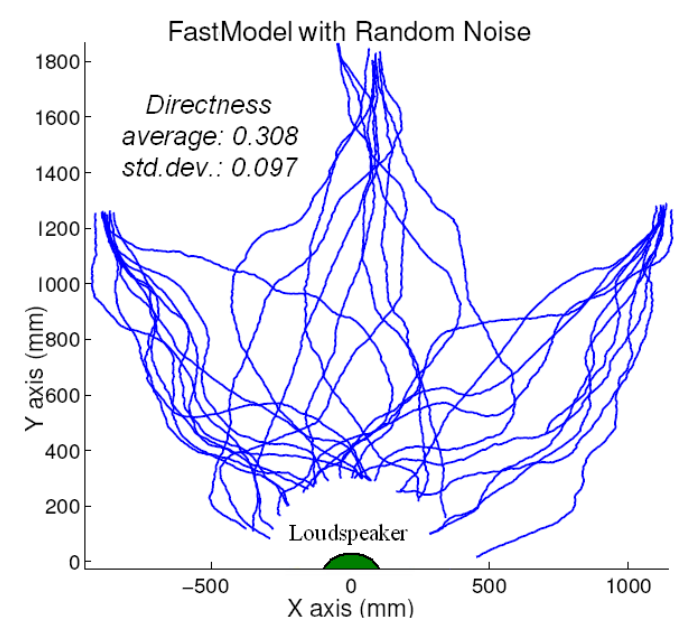

Fig. 7. The Fast model alone makes the robot able to reach the target also when a noise disturbs motor controllers but the robot trajectory are not straighter.

the forward model are verified when a random noise is introduced in the motor system (Fig. 10) The prediction of the phonotactic turns is efficient: in fact the attempts look like the previous experiment when no disturbance was considered. The optomotor reflex is able to compensate turns due to random noise, without conflicting with turns caused by phonotaxis. It means that phonotaxis is able to accomplish its purpose turning the robot towards the sound source. The value of directness is lower than the experiments without noise (directness $=0.34$ ), but improved in respect to the experiments with noise in which the phonotaxis and optomotor behaviours were not integrated by the forward model.

\section{CONCLUSIONS}

In this paper a nonlinear feedforward compensator was designed to solve the problem of multisensory integration in a complex neural network based on the cricket neural system for phonotaxis and optomotor control. The feedfor- 


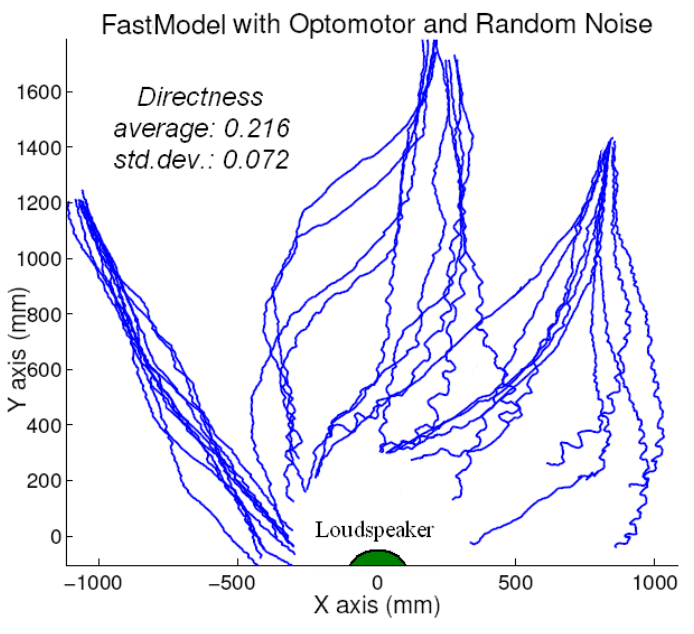

Fig. 8. Optomotor reflex and random noise are enabled and are in conflict with the phonotaxis.

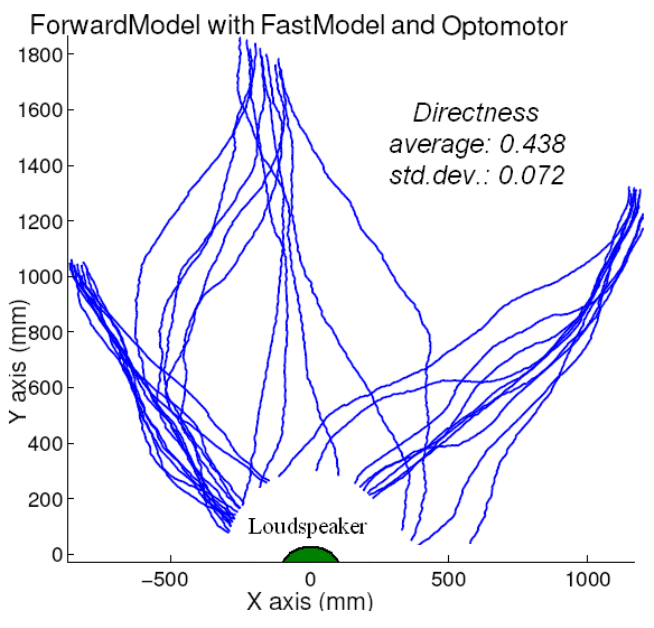

Fig. 9. Forward model correctly integrates phonotaxis and optomotor systems. The robot every time reaches the target.

ward compensation was more efficient than the previous solutions, which simply inhibited optomotor response when a phonotactic induced reflex was taking place. Rather, the implemented feedforward controller showed the capability to correctly predict and filter out phonotactic induced optical noise, leaving the optomotor system ready to react to unknown disturbances, as observed in experiments with crickets. Because the feedforward compensator could be implemented in a realistic spiking neural network, it is a plausible mechanism to explain real cricket behaviour, and shows how biological systems might implement, and tune, this form of control. The result obtained in this paper also opens the way to the hardware design and implementation of the whole neural-controller system for the integration in a complete autonomous machine.

ACKNOWLEDGMENTS: The authors acknowledge the support of the European Commission under the Project FP62003-IST2 - 004690 SPARK. Thanks to Reid Harrison for design of the optomotor sensor chip.

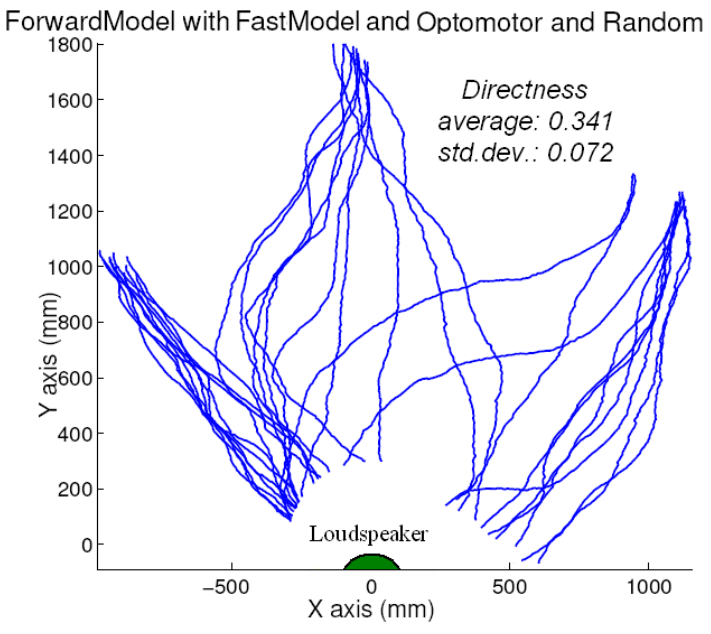

Fig. 10. Noise are enabled. Forward model correctly integrates phonotaxis and optomotor systems. The robot every time reaches the target (phonotaxis), while optomotor compensates the noise.

\section{REFERENCES}

[1] A.I. Selverston, H.U. Kleindienst, F. Huber, "Synaptic Connectivity Between Cricket Auditory Interneurons as Studied by Selective Photoinactivation", The Journal of Neuroscience 5(5):1283-1292, 1985.

[2] K. Imaizumi, G.S. Pollack, "Neural Coding of Sound Frequency by Cricket Auditory Receptors", The Journal of Neuroscience, 19(4):1508-1516, February, 1999.

[3] A. Nabatiyan, J.F.A. Poulet, G.G. de Polavieja, B. Hedwig, "Temporal Pattern Recognition Based on Instantaneous Spike Rate Coding in a Simple Auditory System", J. Neurophysiol. 90:2484-2493, 2003.

[4] H.H. Lund, B. Webb, J. Hallam, "A Robot Attracted to the Cricket Species Gryllus bimaculatus", Fourth European Conference on Artifcial Life 246-255, Cambridge MA, MIT Press, 1997.

[5] H.H. Lund, B. Webb, J. Hallam, "Physical and Temporal Scaling Considerations in a Robot Model of Cricket Calling Song Preference", Artificial Life 4(1):95-107, 1998.

[6] B. Webb, T. Scutt, "A Simple Latency Dependent Spiking Neuron Model of Cricket Phonotaxis", Biological Cybernetics 82(3):247-269, 2000.

[7] R.E. Reeve, B. Webb, "New Neural Circuits for Robot Phonotaxis", Philosophical Transactions of the Royal Society A 361:2245-2266, 2003.

[8] A.D. Horchler, R.E. Reeve, B. Webb, R.D. Quinn, "Robot Phonotaxis in the Wild: a Biologically Inspired Approach to Outdoor Sound Localization", Proc. 11th Int Conf. Advanced Robotics, 2003.

[9] B. Hedwig, J.F.A. Poulet, "Complex Auditory Behaviour Emerges From Simple Reactive Steering", Nature, 430:781-785, 2004.

[10] P. Russo, "Sistemi Neurali Biologici E Controllo Predittivo Per L'Integrazione Acustico-Visiva Nel Grillo", Graduation thesis, Faculty of Computer Science Engineer of Catania, 2005.

[11] B. Webb, R.R. Harrison "Integrating sensorimotor systems in a robot model of cricket behavior", Sensor Fusion and Decentralized Control in Robotic Systems III 4196:113-124, 2000.

[12] B. Webb, R.E. Reeve, "Reafferent or Redundant: How Should a Robot Cricket Use an Optomotor Reflex?", Adaptive Behavior 11(3):137$158,2003$.

[13] B. Webb, "Neural mechanisms for prediction: do insects have forward models?", Trends in Neurosciences, 27:278-282, 2004.

[14] C. Koch, Biophysics of Computation, Oxford University Press, 1999.

[15] R.E. Reeve, "Neural Model Used for Robot Control", Centre for Cognitive and Computational Neuroscience, University of Stirling, 2004.

[16] J.L. Schul, "Song recognition by temporal cues in a group of closely related bushcricket species (genus Tettigonia)", Journal of Comparative Physiology A 183:410-410, 1998. 